\title{
PREFACE
}

\section{A special issue on Optoelectronics in Australia}

\author{
Gang-Ding PENG (凶) \\ Photonics and Optical Communications, School of Electrical Engineering and Telecommunications, \\ University of New South Wales (UNSW) Sydney, 2052, Australia
}

(C) Higher Education Press and Springer-Verlag GmbH Germany, part of Springer Nature 2018

I am very pleased to be the guest editor of the special issue, at the invitation by Frontiers of Optoelectronics. I thank Frontiers of Optoelectronics for providing us the great opportunity to present the latest Australian research in this special issue before a global optoelectronics audience.

The achievements and accomplishments made in the fields of photonics and electronics have greatly transformed our way of living. Optoelectronics, which is closely related to both photonics and electronics, is one of the fastest growing fields and has become an indispensable part of our modern life. Australian researchers have been very actively engaging research and development in photonics, electronics as well as optoelectronics. This issue aims at introducing some of the latest photonics and optoelectronics research in Australia and facilitating the building up of collaboration and partnerships between Australian and international researchers.

This special issue contains 7 review and research papers by Australian researchers. They are on several individual research topics and can offer only a glimpse of the current research and development in several specific topical areas in Australia.

Sincerely I would thank all authors for their scientific contributions and for making special issue really special one! Finally I would express my gratitude to the support of the editors and staff of Frontiers of Optoelectronics.

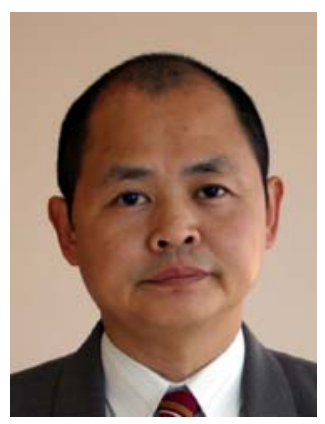

Prof. Gang-Ding Peng received his B.Sc. degree in physics from Fudan University, Shanghai, China, in 1982, and M.Sc. degree in applied physics and Ph.D. degree in electronic engineering from Shanghai Jiao Tong University, Shanghai, China, in 1984 and 1987, respectively. From 1987 through 1988, he was a lecturer of Shanghai Jiao Tong University. He was a postdoctoral research fellow in the Optical Sciences Centre of the Australian National University, Canberra, from 1988 to 1991. He has been working with UNSW since 1991, was a Queen Elizabeth II Fellow from 1992 to 1996 and is currently a Professor in the same university. He is a fellow and life member of both OSA and SPIE. His research interests include specialty silica and polymer optical fibres, optical fibre and waveguide devices, optical fibre sensors and nonlinear optics. So far, he has published more than 300 refereed journal papers and more than 200 conference papers, and co-authored more than 10 book chapters on these subjects.

Email: g.peng@unsw.edu.au

Received January 3, 2018

E-mail: g.peng@unsw.edu.au 